Article

\title{
Fabrication of Modified Random Phase Masks with Phase Modulation Elements Exhibiting Gaussian Profiles Using Molecular Migration under Photopolymerization
}

\author{
Akira Emoto ${ }^{1, *, \dagger}$, Junya Honda ${ }^{1}$, Kou Suzuki ${ }^{1}$, Takumi Kimoto ${ }^{1}$ and Takashi Fukuda ${ }^{2}$ D \\ 1 Graduate School of Science and Engineering, Doshisha University, 1-3 Tatara-miyakodani, Kyotanabe, \\ Kyoto 610-0321, Japan; bup2013@mail4.doshisha.ac.jp (J.H.); bup3082@mail4.doshisha.ac.jp (K.S.); \\ ctwb0317@mail4.doshisha.ac.jp (T.K.) \\ 2 National Institute of Advanced Industrial Science and Technology (AIST), 1-1-1 Higashi Tsukuba, \\ Ibaraki 305-8565, Japan; t-fukuda@aist.go.jp \\ * Correspondence: emoto.akira@tokushima-u.ac.jp; Tel.: +81-886-56-8026 \\ + Present Affiliation: Institute of Post-LED Photonics, Tokushima University, 2-1 Minami-Josanjima, \\ Tokushima, Tokushima 770-8506, Japan.
}

Received: 2 May 2019; Accepted: 28 May 2019; Published: 3 June 2019

\begin{abstract}
Random phase masks are important technical elements for realizing holographic memory systems that enable high density recording. However, the broadly distributed Fourier spectrum often presents a problem because wide recording spots result in reduced total storage capacity for a recording medium. In the present study, we propose modified random phase masks with phase modulation elements exhibiting Gaussian profiles to suppress the spread of the recording spot and keep it in a narrow area, based on the reduction of the high-frequency components in a random phase pattern. We confirm the effectiveness of the proposed random phase mask using simulations of a computer-generated binary hologram. However, issues still remain in terms of the fabrication of random phase masks with Gaussian profiles. Therefore, we evaluate the feasibility of fabricating the proposed random phase mask using molecular diffusion under photopolymerization. The results confirm the feasibility of this approach over a relatively wide area for actual fabrication.
\end{abstract}

Keywords: holographic memory; random phase mask; photopolymer; molecular diffusion

\section{Introduction}

Currently, the global information society exhibits an expansion of data-centric management based on big data analysis. This trend is inevitably linked to a requirement of increased storage capacity for memory devices. Hologram memory is a promising candidate for this purpose because of the use of parallel data processing for recording and the reconstruction of page data at high densities [1-3]. Therefore, numerous related technologies have been studied for optical systems, including recording media [4-13].

Among these technologies, an optical configuration for collinear holography has been proposed [14-19]. In this system, the signal beam with page data is co-axially surrounded by a ring-shaped reference beam. Both beams are simultaneously focused on a recording medium by a lens. As a result, a Fourier hologram can be recorded using a fully integrated optical interferometry system, which enables tracking the surface of a rotational recording medium such as a rewritable optical disk system. With the development of such robust optical systems, studies on various configurations have been reported such as phase modulation masks and apertures for practical 
holographic memory systems $[7,20-25]$. We have investigated the optimal configuration of phase modulation and apertures for Fourier hologram recording and reconstruction [26,27]. Among these studies, we have proposed some optimized apertures to restrict the Fourier spectrum and reduce the recording spot area. However, issues remain on the use of apertures to restrict the Fourier image distribution. If placed close to the recording medium, the aperture will be in contact with the surface of the medium under high-speed rotation. If the aperture is placed far from the surface of the recording medium, one must then consider the influence of diffraction at the aperture edge. It would be preferable to achieve a narrow Fourier spot without introducing an aperture. On the basis of this consideration, we previously proposed advanced random phase masks [28,29]. However, the reconstruction quality has not yet reached the level required for practical holographic memory systems. The goal of our subsequent studies is to develop a random phase mask that generates a relatively narrow Fourier image with a moderate quality of page data reconstruction.

In this study, we propose a modified random phase mask with phase modulation elements exhibiting Gaussian profiles to restrict the distribution to a narrow area based on the reduction of high-frequency components in the random phase modulation pattern. We have preliminarily confirmed the effectiveness of these modified random phase masks; benefits were observed for both the confinement of the Fourier image distribution and the reconstruction quality. However, it is difficult to fabricate artificial random phase masks with Gaussian profiles using conventional micro-fabrication processes such as photolithographic method based on the etching process [30]. Thus, our primary purpose is to evaluate the feasibility of producing random phase masks with gradual modulations, such as Gaussian profiles. For this purpose, we employed molecular diffusion under photopolymerization to obtaining the proposed random phase masks. Molecular migration under photopolymerization has been studied well [31-36]. In the initial stages, molecular migration is induced toward a bright region, which results in the formation of a smooth convex structure [34,35]. Using this formation, the feasibility of fabricating random phase masks with phase modulation elements exhibiting Gaussian profiles has been confirmed over a relatively wide area.

\section{Preliminary Investigation on the Effectiveness of the Proposed Random Phase Mask}

To estimate the effectiveness of the proposed random phase mask, the reconstruction quality of a computer-generated binary hologram (CGBH) was simulated using a principle-based algorithm for a Lohmann-type binary hologram [37-39]. Random phase modulation can be introduced in the simulation, and the reconstruction quality can be systematically evaluated. Therefore, this is a suitable approach for examining the effectiveness of modified random phase patterns [29].

We considered three types of random modulation patterns, as shown in Figure 1. Here, amplitude modulations were used to understand their fundamental spatial frequencies in the three types of random modulation patterns. The first is a typical binary modulation pattern that includes numerous high spatial frequencies (Figure 1i). The Fourier image is broadly distributed as a two-dimensional sinc function. The Fourier image distribution can be confined within a relatively narrow area using a Gaussian-like modulation (Figure 1ii). However, if some modulation elements are placed adjacently, cross-sectional distribution differs from that of conventional binary modulation. In addition, the isolated modulation generates a certain periodicity, which can be confirmed as spike-like distributions in the Fourier image. To suppress the generation of spikes, continuous Gaussian-like modulation was introduced (Figure 1iii). The cross-sectional distribution exhibits a Gaussian profile only at the edges. Thus, inhibition of the spike-like distribution in the Fourier image was confirmed. 


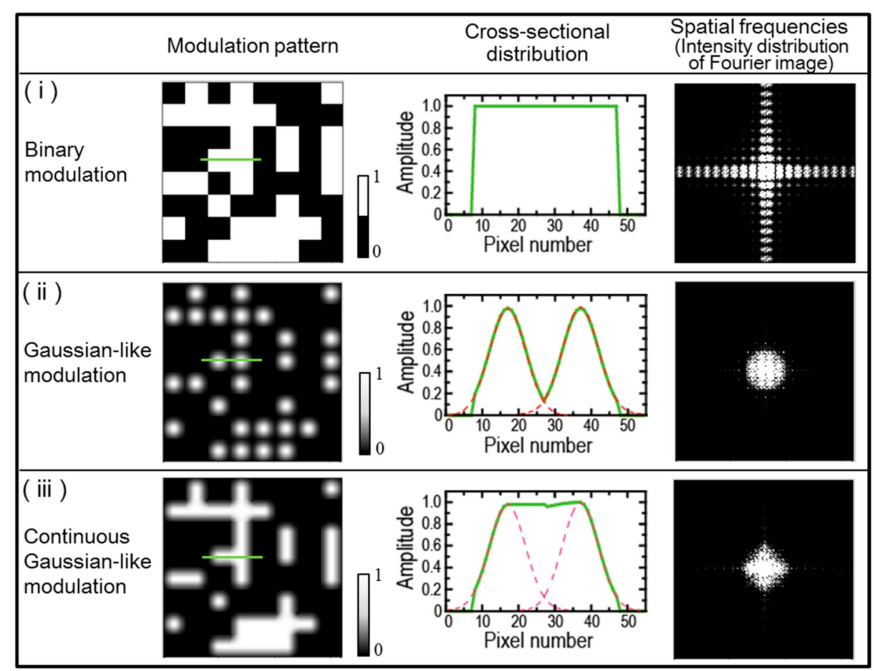

Figure 1. Three types of two-dimensional random modulation patterns.

The fundamental features of these three types of two-dimensional random modulation patterns were introduced for the random phase modulation in hologram recordings of a binary image. A binary image of " $\mathrm{ABC}$ " was used to evaluate the effectiveness of each random phase pattern, as shown in Figure 2. The binary image of "ABC" $(120 \times 60$ pixels $)$ was placed on a part of the signal image ( $256 \times 256$ pixels). The simulation was performed using Lohmann-type CGBH algorithm [29]. The cell size (window size) of the Lohmann hologram was set at $9 \times 9$ pixels. Therefore, the resultant hologram size was $2304 \times 2304$ pixels. Finally, the CGBH was reconstructed by an inverse-Fourier transformation, and the corresponding area was extracted to evaluate the reconstruction quality. When no random phase modulation was added to the original binary image, the CGBH generated from the Fourier-transformation result of the original image was narrowly distributed, corresponding to a small recording spot, but the reconstruction quality is extremely poor, as expected (Figure 2A). The reconstruction quality is substantially improved by adding a high-resolution random phase modulation, as shown in Figure 2B. However, the CGBH generated from the Fourier image is broadly distributed, which results in a larger recording spot and a reduced storage capacity for the recording medium. As a simple solution, the distribution can be narrowed using a relatively low-resolution random phase; however, the reconstruction quality is degraded again, as shown in Figure 2C. (Here, the phase modulation element consisted of $10 \times 10$ pixels). These results demonstrate the fundamental characteristics of Fourier hologram recording and reconstruction. We proposed a Gaussian-like phase modulation element in random phase modulation, as shown in Figure 2D. A relatively small CGBH distribution was obtained, but the reconstruction quality was still poor, similar to that for low-resolution random phase modulation. We modified the Gaussian-like modulation to continuous Gaussian-like modulation, and the reconstruction quality was significantly improved while maintaining a relatively small CGBH distribution. In this case, the signal-to-noise ratio (SNR) and root-mean-square error (RMSE) were improved, second only to that of the high-resolution random phase modulation. This trend was also observed in the average values obtained from ten samples of each of the four types of random phase patterns in Figure 2, as shown in Figure 3. (Here, the hologram area was determined as the summation of white pixel numbers in each CGBH). More specifically, a reduced RMSE, increased SNR, and confined hologram area (recording spot) were obtained using the continuous Gaussian-like random phase modulation pattern for the Fourier hologram, despite its relatively low-resolution random phase pattern. 


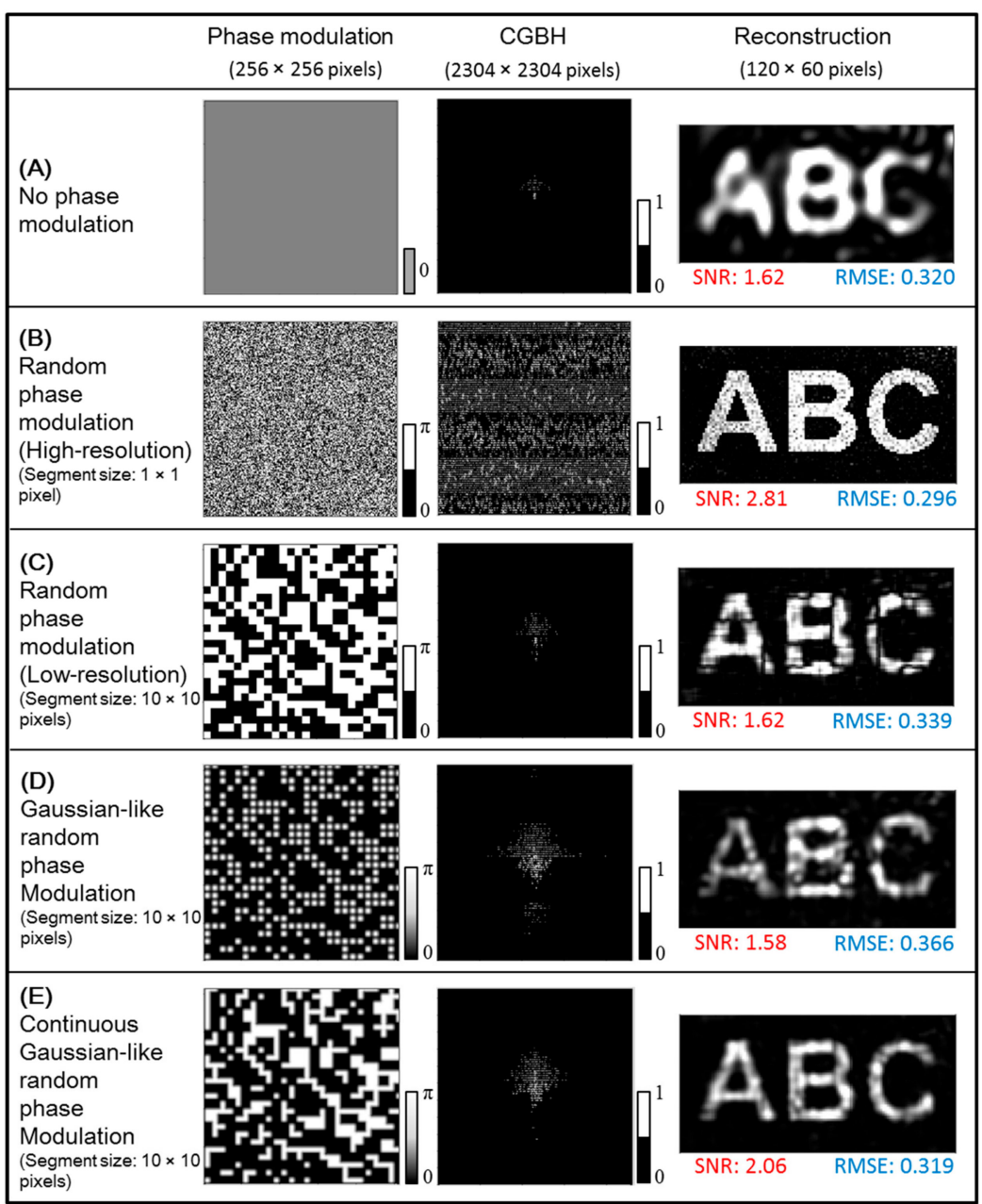

Figure 2. Various random phase patterns, computer-generated binary hologram (CGBH) distributions, and reconstruction results.

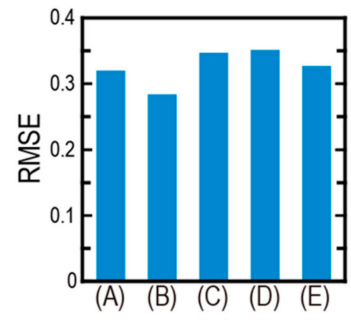

(a)

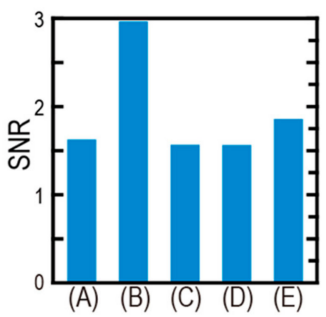

(b)

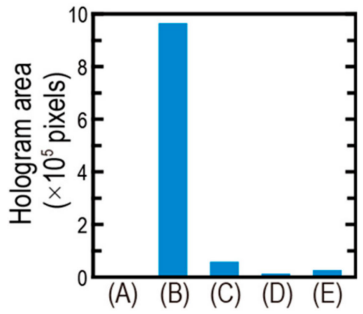

(c)

Figure 3. Comparison of the (a) root-mean-square error (RMSE), (b) signal-to-noise ratio (SNR), and (c) hologram area of the CGBHs. The data labels of (A)-(E) correspond to the labels in Figure 2.

The need to determine the feasibility of fabricating these random phase masks originated from these preliminary investigations.

\section{Materials and Methods}

To induce a smooth surface volume change, we employed molecular migration based on diffusion under photopolymerization. We prepared a photopolymer film on a glass substrate by spin-coating a commercial photopolymer material (NOA 81, Norland Products, Inc. (Cranbury, NJ, USA)). The pre-polymer liquid is polymerized by photoirradiation, based on a thiol-ene reaction [40]. Film thickness can be controlled using the rotation speed. For rotation speeds of 6000,4000 , and $2000 \mathrm{rpm}$, film thicknesses of 4, 6, and $9 \mu \mathrm{m}$ were obtained, respectively. Molecular migration can be induced 
by polymerization upon the irradiation of spatially modulated ultraviolet (UV) light, as shown in Figure $4[34,35,41]$. In photopolymerizable materials, molecular migration occurs in two phases. The first phase corresponds to the initial stage of polymerization, resulting in a convex surface structure. In this stage, the monomers are assembled (or diffused) toward the bright region because the monomer density is reduced by the polymerization in that region. In the second stage, advanced polymerization eliminates unreactive molecules from the bright region, which results in the formation of concave surface structures. As a result, surface volume changes occur, as illustrated in Figure 4.

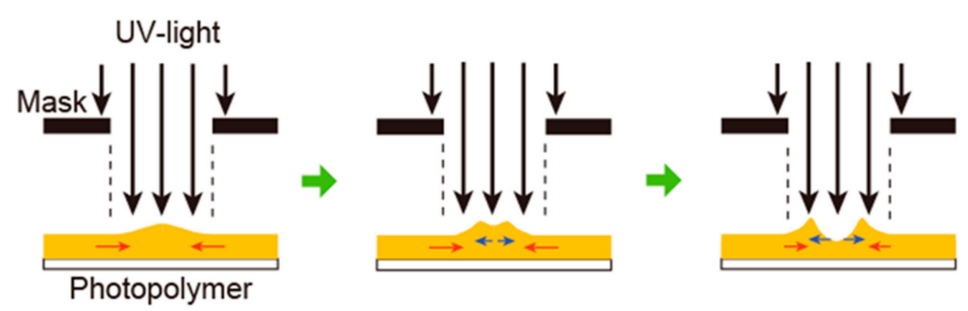

Figure 4. Surface volume changes in the photopolymer material film.

To compare the formation characteristics with those of the other photoreactive materials without molecular migration, we prepared a functional polyvinyl alcohol (PVA, Biosurfine AWP-MRH, Toyo Gosei Co., Ltd. (Tokyo, Japan)). This PVA includes a photoreactive azide group in the side chain. When exposed to UV light, the azide groups are activated and crosslinked to peripheral sites, such as $\mathrm{C}-\mathrm{H}$ bonding or double bonding structures [42-44]. This photo-crosslinking induce water insolubility corresponding to the UV irradiation pattern, which enables typical photo-patterning with water-rinsing. Similar to the preparation of the NOA 81 film, the PVA was spin-coated on a glass substrate, with a film thickness of approximately $1.4 \mu \mathrm{m}$.

The prepared films of NOA 81 were irradiated by UV light with random phase patterns using an optical system, as shown in Figure 5a. UV light with a wavelength of $365 \mathrm{~nm}$, emitted from an LED, was spatially modulated by a digital mirror device (DMD, DLP6500, Texas Instruments, Inc. (Dallas, TX, USA)), and the modulation pattern of the UV light was irradiated on the films via an imaging lens system. The power density of the UV irradiation was set at $11.1 \mathrm{~mW} / \mathrm{cm}^{2}$. The conditions of irradiation time are described in Section 4.2 including the discussion of the influence. After irradiation, the film was exposed to homogenous UV irradiation to complete the photoreaction of the entire photopolymer film. The power density and irradiation time were $60 \mathrm{~mW} / \mathrm{cm}^{2}$ and $120 \mathrm{sec}$, respectively. For this process, surface volume changes were observed using a laser microscope (VK-X210, Keyence Co. (Osaka, Japan)).
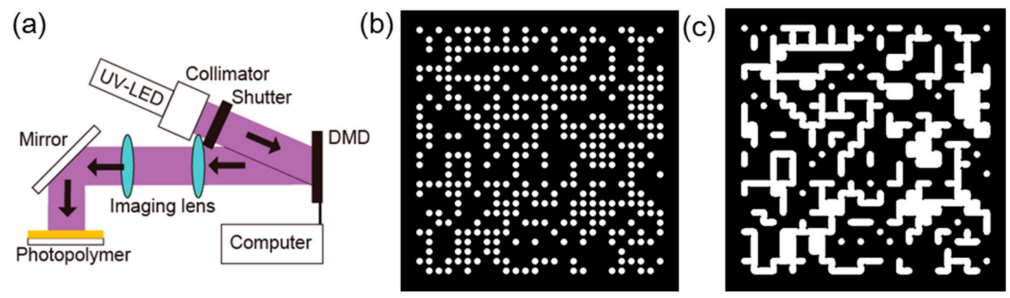

Figure 5. (a) Optical setup for pattern irradiation and irradiation patterns for (b) Gaussian-like and (c) continuous Gaussian-like random phase masks.

To fabricate the Gaussian-like and continuous Gaussian-like random phase masks using molecular migration under photopolymerization, irradiation patterns were prepared, as shown in Figure 5b,c, respectively. The irradiation patterns correspond to binary modulations of optical intensity. Surface volume changes are induced gradually and smoothly, as shown in Figure 4, under the binary modulation of UV irradiation; thus, it was expected that phase modulation elements with Gaussian profiles would form on the photopolymer films. In the present study, we evaluate the 
feasibility of this concept in terms of the formation of phase modulation masks with smooth profiles based on surface volume changes associated with molecular diffusion under photopolymerization.

\section{Results and Discussion}

\subsection{Conventional Fabrication of a Random Phase Mask}

A random phase mask was photo-patterned using a modified PVA film spin-coated on a glass substrate. As described in Section 3, no surface volume changes are induced in this material. A photo-crosslinking of azide moieties is generated by UV irradiation, which makes the polymer insoluble in water. We performed photo-patterning using the binary modulation pattern shown in Figure 6a by applying a power density of $25 \mathrm{~mW} / \mathrm{cm}^{2}$ to the PVA film for $5 \mathrm{~s}$, followed by a water rinsing step. As a result, structural modulations corresponding to the irradiation pattern were observed, as shown in Figure 6b,c. Figure 6d shows the cross-sectional distribution of the patterned PVA film. Typical formation of the photo-patterned film was confirmed, similar to that obtained by photolithography using photo-resist materials. In this case, the bottom substrate was exposed in the valley region, since the bottom layers are relatively flat. At the top surface (hill region), the height is aligned at a constant level, indicating that no surface volume change was induced under photoreaction in the PVA film.
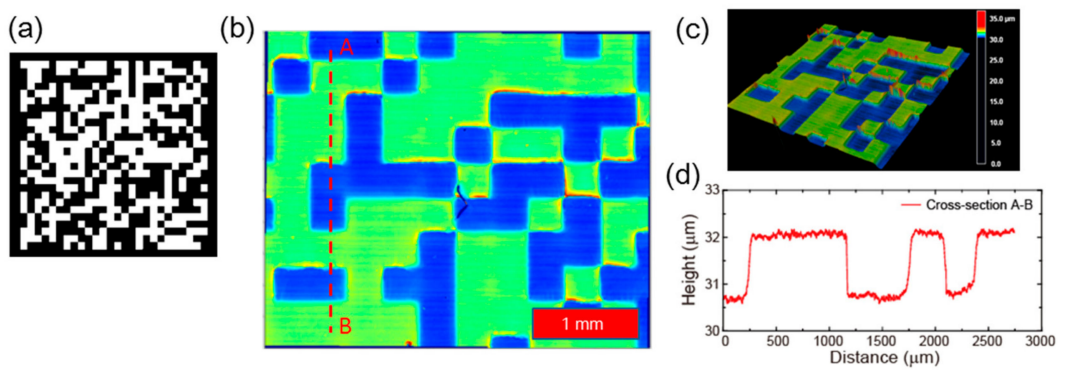

Figure 6. (a) Binary irradiation pattern, (b) top view and (c) three-dimensional image of the patterned PVA film, and (d) cross-sectional distribution of the volume changes.

On the basis of this experiment, we also examined the optical system shown in Figure 5a. The edges of the patterned PVA were relatively sharp. Thus, this optical patterning system can be used to fabricate the proposed random phase masks.

\subsection{Gaussian-Like Random Phase Modulation Mask}

As a second experiment, we fabricated a random phase mask with phase modulation elements exhibiting Gaussian-like profiles using the irradiation pattern shown in Figure 5b for a photopolymer film with surface volume changes induced by photopolymerization. Each phase modulation element was patterned using circular irradiation areas. Therefore, an isolated convex structure would form in the bright region with the smooth shape of the Gaussian-like convex structure during the initial stage of molecular migration, as illustrated in Figure 4. It was expected that UV irradiation time would be an important factor in the fabrication of a phase modulation element with an ideal Gaussian profile, because the surface shape of the photopolymer film changes depending on the UV irradiation time. (Then, the UV irradiation must be stopped when the surface shape is close to Gaussian profiles.) In addition, it has been reported that the initial film thickness is another important factor influencing the characteristics of surface volume changes [34]. Therefore, we varied both the irradiation time for the photo-patterning step and the initial film thickness for the fabrication of a random phase mask with Gaussian-like phase modulation elements.

The resultant surface structures were observed by laser microscopy, as shown in Figure 7. We can see that the relatively thin film is superior for the formation of isolated Gaussian-like convex structures. Moreover, we can also see that a longer irradiation time is preferred for the formation of a Gaussian 
profile. (Here, the fringe-like modulation superimposed on the cross-sectional distribution in Figure 7 is due to the interference effect in laser microscopy). Next, we focused on the results shown in Figure 7d,g. Both samples were formed using photopolymer films with a thickness of $4 \mu \mathrm{m}$. The samples are close to the ideal convex structure, as outlined by Gaussian curves (indicated by the red lines in Figure 7d,g) in their cross-sectional distributions.

Furthermore, the desired isolation between adjacent convex structures was also obtained, as indicated by the red arrows in the cross-sectional distributions in Figure $7 \mathrm{~d}$. Therefore, we determined that a 4 - $\mu \mathrm{m}$-film-thickness and a 2-s-irradiation time are suitable for fabricating a random phase mask with phase modulation elements exhibiting Gaussian-like profiles in these experiments. Further precise investigations of the fabrication parameters, including the height of the convex structure needed to obtain the desired phase shift for the objective wavelength, are needed as a future work.

The viscosity of the photopolymer material used in this study is $300 \mathrm{cps}$ at $25^{\circ} \mathrm{C}$. If another material with a lower viscosity can be introduced or if the viscosity of this photopolymer material can be reduced, the formation characteristics can be controlled, and more preferable conditions can be employed to produce the desired Gaussian modulation.

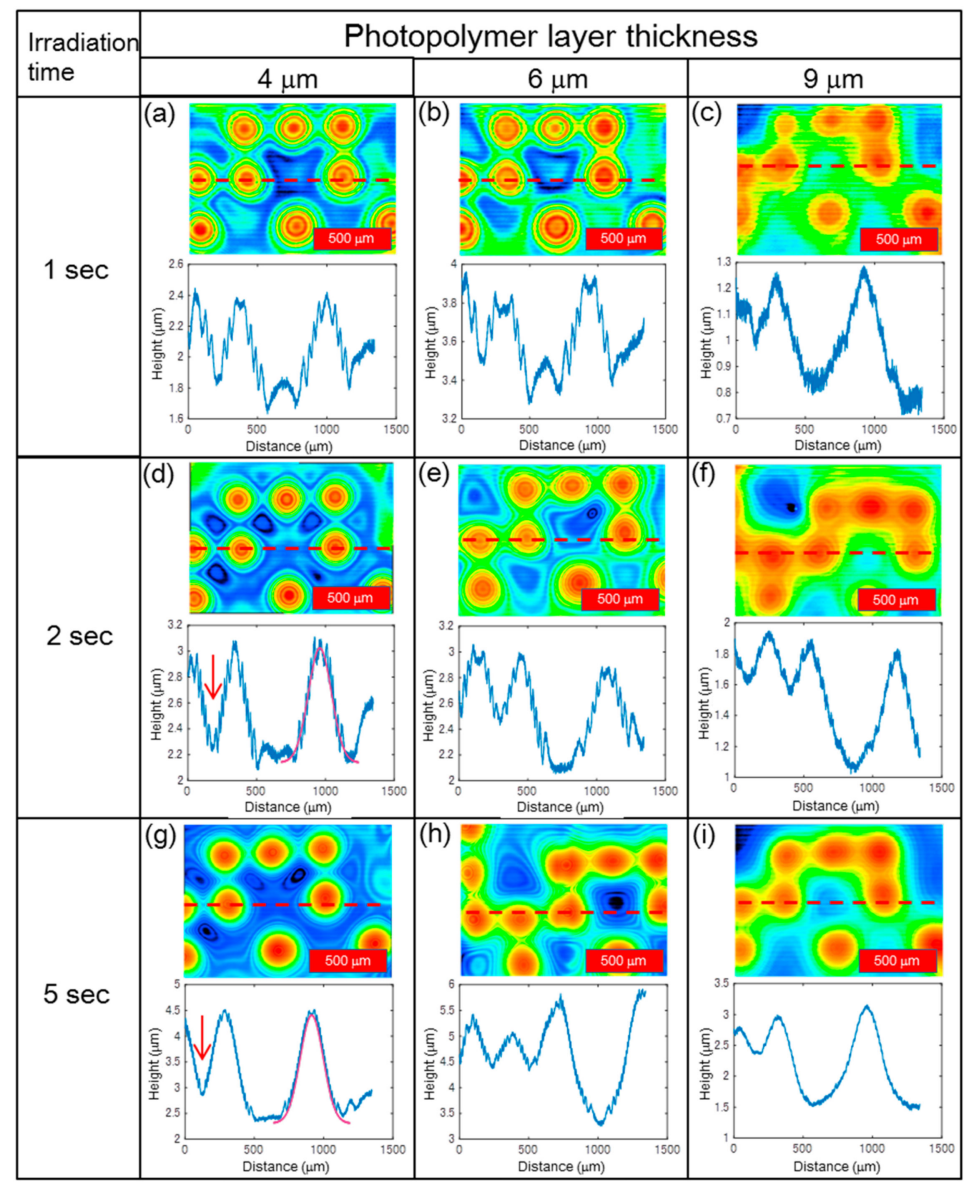

Figure 7. Two-dimensional surface volume changes and the cross-sectional distributions for Gaussian-like random phase masks under photopolymerization depending on both the photopolymer layer thickness and UV irradiation time.

\subsection{Continuous Gaussian-Like Random Phase Modulation Mask}

We also fabricated another random phase mask with phase modulation elements exhibiting continuous Gaussian-like profiles using the irradiation pattern shown in Figure 5c for the photopolymer film, similar to the case for the Gaussian-like random phase element. Each phase modulation element was continuously formed in contrast to the process for the isolated Gaussian-like random phase mask. 
This approach is also different from the typical case of a conventional random phase mask, such as the irradiation pattern shown in Figure 6a. Each corner of the irradiation pattern is rounded because a smooth Gaussian profile is needed for each cross-section of the resultant random phase mask. For the experimental conditions, we varied both the photo-patterning irradiation time and the initial film thickness for the fabrication of a continuous Gaussian-like random phase mask, similar to the case of the Gaussian-like random phase mask.

The resultant surface structures are shown in Figure 8. Clearly, the film with an intermediate thickness of approximately $6 \mu \mathrm{m}$ is preferred for the formation of a homogenous Gaussian-like convex structure. In addition, a longer irradiation time is preferred for the formation of a smooth Gaussian profile, similar to the case of the Gaussian-like random phase mask. Here, we focused on the results shown in Figure 8d-f. We can see that the top portions of the phase modulation elements (i.e., the top portions of the hills indicated by red arrows) exhibit different structures, depending on the initial thickness of the photopolymer layer. Concave, flat, and convex shapes were obtained for the top portions of the patterned films with initial thicknesses of 4,6 , and $9 \mu \mathrm{m}$, respectively. The edge shape of the cross-sectional distribution in Figure $8 \mathrm{e}$ is relatively close to the Gaussian distributions, as indicated by a red line for each distribution. Therefore, for the formation of an ideal continuous Gaussian-like random phase mask, a film thickness of $6 \mu \mathrm{m}$ and an irradiation time of $2 \mathrm{~s}$ are ideal.

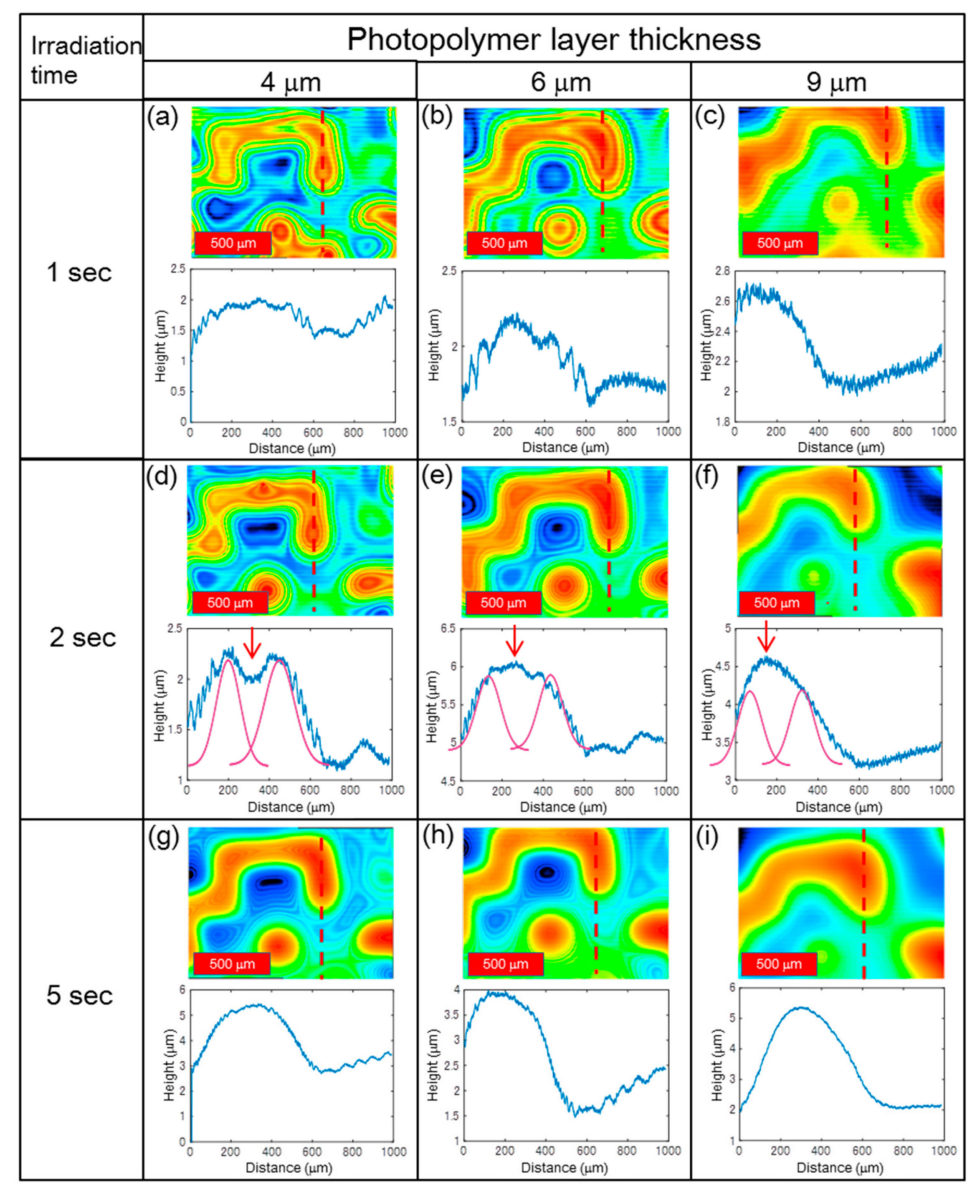

Figure 8. Two-dimensional surface volume changes and the cross-sectional distributions for continuous Gaussian-like random phase masks under photopolymerization depending on both the photopolymer layer thickness and UV irradiation time.

\subsection{Large-Area Formation}

To realize these advanced random phase masks for practical hologram memory systems, the feasibility of homogenous large-area formation must be also considered. We measured the surface 
volume changes over a wide area $\left(\sim 10 \times 10 \mathrm{~mm}^{2}\right)$ for Gaussian-like and continuous Gaussian-like random phase masks formed under the optimized conditions described in Sections 4.2 and 4.3, respectively, as shown in Figure 9. These measurements were performed using the sequential stage control system of the laser microscope. Clearly, the formation of the phase modulation masks is homogenous. Moreover, ideal formations were observed for the random phase masks with phase modulation elements exhibiting continuous Gaussian-like profiles.

(a)

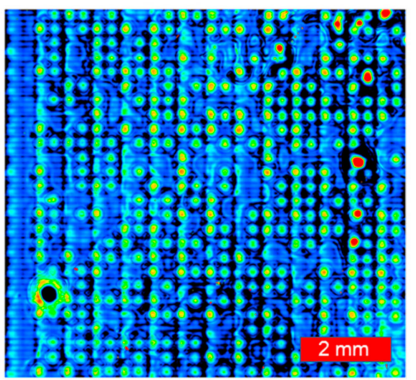

(c)

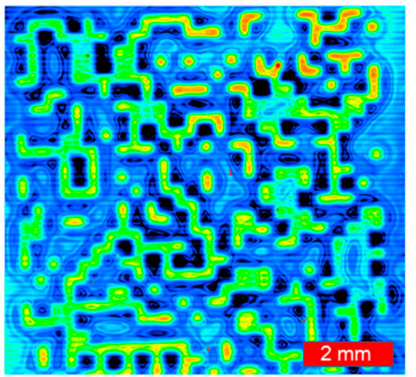

(b)

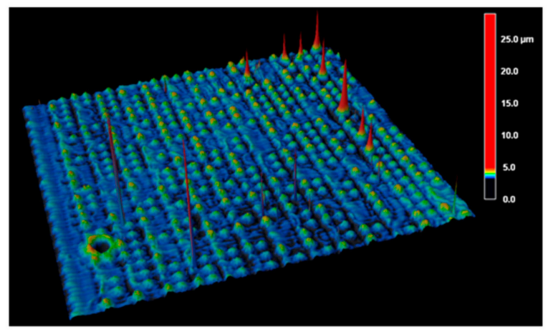

(d)

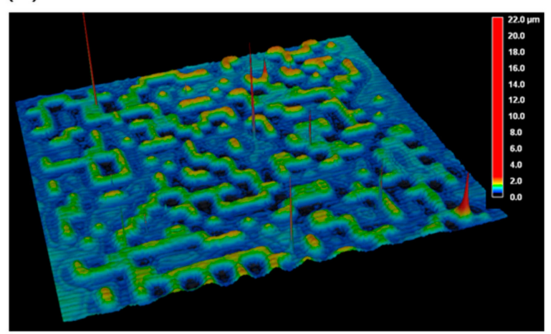

Figure 9. Wide-area measurement of surface modulations for $(\mathbf{a}, \mathbf{b})$ Gaussian-like and $(\mathbf{c}, \mathbf{d})$ continuous Gaussian-like random phase masks.

\section{Conclusions}

We have demonstrated the advantages of a random phase modulation pattern with phase modulation elements exhibiting continuous Gaussian-like profiles based on a preliminary investigation of CGBH simulations. The feasibility of fabricating these masks for practical use was evaluated. Photo-patterned surface volume changes induced by molecular migration under photopolymerization were employed for the fabrication. The results show that Gaussian-like convex structures and corresponding arrays can be formed with relatively high quality. The essential factors and conditions for forming ideal structures were also determined. In a future work, full-fledged investigations are needed to realize these random phase masks, including an exploration of their hologram recording characteristics. We expect that the proposed random phase mask will be a useful optical element for hologram memory systems.

Author Contributions: A.E. and T.F. conceived and designed the experiments; J.H. and A.E. prepared the random phase patterns; J.H., K.S., and T.K. performed the experiments and analyzed the data; A.E. wrote the paper.

Funding: This research was partially funded by the Japan Science and Technology Agency (JST) under the Strategic Promotion of Innovative Research and Development Program.

Conflicts of Interest: The authors declare no conflict of interest.

\section{References}

1. Hill, B. Some aspects of a large capacity holographic memory. Appl. Opt. 1972, 11, 182-191. [CrossRef]

2. Pu, A.; Psaltis, D. High-density recording in photopolymer-based holographic three-dimensional disks. Appl. Opt. 1996, 35, 2389-2398. [CrossRef] 
3. Ashley, J.; Jefferson, C.M.; Bernal, M.-P.; Marcus, B.; Burr, G.W.; Macfarlane, R.M.; Coufal, H.; Shelby, R.M.; Guenther, H.; Sincerbox, G.T.; et al. Holographic data storage. IBM J. Res. Develop. 2000, 44, 341-368. [CrossRef]

4. Takeda, Y.; Oshida, Y.; Miyamura, Y. Random phase shifters for Fourier transformed holograms. Appl. Opt. 1972, 11, 818-822. [CrossRef]

5. Mok, F.H. Angle-multiplexed storage of 5000 holograms in lithium niobate. Opt. Lett. 1993, 18, 915-917. [CrossRef] [PubMed]

6. Psaltis, D.; Levene, M.; Allen, P.; Barbastathis, G. Holographic storage using shift multiplexing. Opt. Lett. 1995, 20, 782-784

7. Hong, J.; McMichael, I.; Ma, J. Influence of phase masks on cross talk in holographic memory. Opt. Lett. 1996, 21, 1694-1696. [CrossRef]

8. Bashaw, M.C.; Hesselink, L. Digital wavelength-multiplexed holographic data storage system. Opt. Lett. 1996, 21, 1780-1782.

9. Gao, Q.; Kostuk, R. Improvement to holographic digital data-storage system with random and pseudorandom phase masks. Appl. Opt. 1997, 36, 4853-4861. [CrossRef]

10. Bruder, F.-K.; Hagen, R.; Rölle, T.; Weiser, M.-S.; Fäcke, T. From the surface to volume: Concepts for the next generation of optical-holographic data-storage materials. Angew. Chem. Int. Ed. 2011, 50, 4552-4573. [CrossRef]

11. Shimobaba, T.; Ito, T. Random phase-free computer-generated hologram. Opt. Express 2015, $23,9549-9554$. [CrossRef] [PubMed]

12. Shimada, K.; Hosaka, M.; Yamazaki, K.; Onoe, S.; Ide, T. Technique for positioning hologram for balancing large data capacity with fast readout. Jpn. J. Appl. Phys. 2017, 56, 09NA04-1-09NA04-8. [CrossRef]

13. Liu, S.; Fu, S.; Zhang, X.; Wang, X.; Kang, L.; Han, X.; Chen, X.; Wu, J.; Liu, Y. UV-resistant holographic data storage in noble-metal/semiconductor nanocomposite films with electron acceptors. Opt. Mater. Express 2018, 8, 1143-1153. [CrossRef]

14. Horimai, H.; Tan, X.; Li, J. Collinear holography. Appl. Opt. 2005, 44, 2575-2579. [CrossRef] [PubMed]

15. Tanaka, K.; Hara, M.; Tokuyama, K.; Hirooka, K.; Ishioka, K.; Fukumoto, A.; Watanabe, K. Improved performance in coaxial holographic data recording. Opt. Express 2007, 15, 16196-16209. [CrossRef]

16. Sun, C.-C.; Yu, Y.-W.; Hsieh, S.-C.; Teng, T.-C.; Tsai, M.-F. Point spread function of a collinear holographic storage system. Opt. Express 2007, 15, 18111-18118. [CrossRef] [PubMed]

17. Yu, Y.-W.; Chen, C.-Y.; Sun, C.-C. Increase of signal-to-noise ratio of a collinear holographic storage system with reference modulated by a ring lens array. Opt. Lett. 2010, 35, 1130-1132. [CrossRef] [PubMed]

18. Nobukawa, T.; Nomura, T. Design of high-resolution and multilevel reference pattern for improvement of both light utilization efficiency and signal-to-noise ratio in coaxial holographic data storage. Appl. Opt. 2014, 53, 3773-3781. [CrossRef]

19. Liu, J.; Horimai, H.; Lin, X.; Huang, Y.; Tan, X. Phase modulated high density collinear holographic data storage system with phase retrieval reference beam locking and orthogonal reference encoding. Opt. Express 2018, 26, 3828-3838. [CrossRef]

20. Ioka, A.; Kurahashi, K. Holographic image formation using phase plates with incoherent imaging property. Appl. Opt. 1976, 15, 1787-1794.

21. Bernal, M.-P.; Burr, G.W.; Coufal, H.; Grygier, R.K.; Hoffnagle, J.A.; Jefferson, C.M.; Oesterschulze, E.; Shelby, R.M.; Sincerbox, G.T.; Quintanilla, M. Effects of multilevel phase masks on interpixel cross talk in digital holographic storage. Appl. Opt. 1997, 36, 3107-3115. [CrossRef] [PubMed]

22. Várhegyi, P.; Koppa, P.; Ujhelyi, F.; Lőrincz, E. System modeling and optimization of Fourier holographic memory. Appl. Opt. 2005, 44, 3024-3030. [CrossRef] [PubMed]

23. Hara, M.; Tanaka, K.; Tokuyama, K.; Toishi, M.; Hirooka, K.; Fukumoto, A.; Watanabe, K. Linear reproduction of a holographic storage channel using coherent addition of optical DC components. Jpn. J. Appl. Phys. 2008, 47, 5885-5890. [CrossRef]

24. Gu, H.; Yin, S.; Tan, Q.; Cao, L.; He, Q.; Jin, G. Improving signal-to-noise ratio by use of a cross-shaped aperture in the holographic data storage system. Appl. Opt. 2009, 48, 6234-6240. [CrossRef] [PubMed]

25. Ishii, T.; Fujimura, R. Interpixel crosstalk cancellation on holographic memory. Jpn. J. Appl. Phys. 2017, 56, 09NA10-1-09NA10-6. [CrossRef] 
26. Nobukawa, T.; Barada, D.; Nomura, T.; Fukuda, T. Orthogonal polarization encoding for reduction of interpixel cross talk in holographic data storage. Opt. Express 2017, 25, 22425-22439. [CrossRef] [PubMed]

27. Takeuchi, S.; Nobukawa, T.; Barada, D.; Fukuda, T.; Emoto, A. Suppression of inter-pixel cross talk and reduction of recording spot size for high-density holographic memory. Opt. Rev. 2019, 26, 124-130. [CrossRef]

28. Emoto, A.; Fukuda, T. Randomly displaced phase distribution design and its advantage in page-data recording of Fourier transform holograms. Appl. Opt. 2013, 52, 1183-1191. [CrossRef]

29. Hiramoto, S.; Fukuda, T.; Emoto, A. Randomly displaced phase distribution design for computer generated binary holograms with narrow recording spots. Opt. Rev. 2018, 25, 509-516. [CrossRef]

30. Burckhardt, C.B. Use of a random phase mask for the recording of Fourier transform holograms of data masks. Appl. Opt. 1970, 9, 695-700. [CrossRef]

31. Croutxé-Barghorn, C.; Lougnot, D.J. Use of self-processing dry photopolymers for the generation of relief optical elements: a photochemical study. Pure Appl. Opt. 1996, 5, 811-825. [CrossRef]

32. Ono, H.; Tamoto, T.; Emoto, A.; Kawatsuki, N. Holographic recording in photoreactive monomer/polym composites. Jpn. J. Appl. Phys. 2005, 44, 1781-1786. [CrossRef]

33. Goldenberg, L.; Sakhno, O.; Stumpe, J. Application of Norland adhesive for holographic recording. Opt. Mater. 2005, 27, 1379-13856. [CrossRef]

34. Sánchez, C.; de Gans, B.-J.; Kozodaev, D.; Alexeev, A.; Escuti, M.J.; van Heesch, C.; Bel, T.; Schubert, U. Polymerization-induced diffusion as a tool to generate periodic relief structures: A combinatorial study. Proc. SPIE 2006, 6136, 61360H-1-61360H-12.

35. Aoki, K.; Ichimura, K. Self-developable surface relief photoimaging generated by anionic UV-curing of epoxy resins. Polym. J. 2009, 41, 988-992. [CrossRef]

36. Emoto, A.; Baharim, S.B.; Sasaki, T.; Shioda, T.; Ogiwara, A.; Ono, H. Chronological investigations of Raman-Nath diffraction grating inscribed by direct laser writing in photoreactive monomer base mixtures. Jpn. J. Appl. Phys. 2010, 49, 122502-1-122502-6. [CrossRef]

37. Lohmann, A.W.; Paris, D.P. Binary Fraunhofer holograms, generated by computer. Appl. Opt. 1967, 6, 1739-1748. [CrossRef]

38. Martinez, C.; Lemonnier, O.; Laulagnet, F.; Fargeix, A.; Tissot, F.; Armand, F. Complementary computer generated holography for aesthetic watermarking. Opt. Express 2012, 20, 5547-5556. [CrossRef]

39. Tamura, H.; Ishii, Y. Computer-generated hologram fabricated by electron-beam lithography for noise reduction. Opt. Rev. 2012, 19, 50-57. [CrossRef]

40. Wägli, Ph.; Homsy, A.; de Rooij, N.F. Norland optical adhesive (NOA81) microchannels with adjustable wetting behavior and high chemical resistance against a range of mid-infrared-transparent organic solvents. Sen. Actuators B 2011, 156, 994-1001. [CrossRef]

41. Baten'kin, M.A.; Mensov, S.N. Optical formation of polymeric materials with heterogeneously distributed nanopores from a photopolymerizable composite. J. Polym. Res. 2015, 22, 64-1-64-8.

42. Mishra, R.K.; Dominguez, R.B.; Bhand, S.; Muñoz, R.; Marty, J.-L. A novel automated flow-based biosensor for the determination of organophosphate pesticides in milk. Biosens. Bioelectron. 2012, 32, 56-61. [CrossRef] [PubMed]

43. Emoto, A.; Noguchi, N.; Kobayashi, T.; Fukuda, T. Fabrication of submicrometer pores with an outer shell using modified poly(vinyl alcohol) and the molecular or particle collection effect. Langmuir 2013, 29, 12601-12607. [CrossRef] [PubMed]

44. Ossipov, D.A.; Hilborn, J. Poly(vinyl alcohol)-based hydrogels formed by "Click Chemistry". Macromolecules 2006, 39, 1709-1718. [CrossRef]

(C) 2019 by the authors. Licensee MDPI, Basel, Switzerland. This article is an open access article distributed under the terms and conditions of the Creative Commons Attribution (CC BY) license (http://creativecommons.org/licenses/by/4.0/). 